\title{
Simultaneous determination of vitamin B6 and catechins in dietary supplements by ZIC-HILIC chromatography and their antioxidant interactions
}

\author{
Aleksandra Sentkowska ${ }^{1} \cdot$ Sylwia Piwowarczyk ${ }^{2} \cdot$ Krystyna Pyrzyńska $^{2}$
}

Received: 12 March 2020 / Revised: 8 May 2020 / Accepted: 16 May 2020 / Published online: 26 May 2020

(c) The Author(s) 2020

\begin{abstract}
Hydrophilic interaction liquid chromatography coupled to mass spectrometry was employed for simultaneous determination of vitamin B6 and catechins in dietary supplements. The obtained results clearly shows the potential of the application of alcohol eluent (instead mainly used acetonitrile) as a component of an with zwitterionic stationary phase. The limits of detection on ZIC-HILIC column were $0.01 \mathrm{mg} / \mathrm{L}$ for catechins and vitamins B (only for pyridoxal phosphate was $0.10 \mathrm{mg} / \mathrm{L}$ ). The investigations between green tea extract (GTE) and vitamin B were also evaluated using isobolographic analysis as well as the interaction indexes. Antioxidant activities of single components and their mixtures were determined by DPPH assay. It was found that the mixtures of GTE and vitamin B acted synergistically. In comparison to GTE alone, faster DPPH radical bleaching of the mixtures was observed in the presence of different forms of vitamin B6 (pyridoxine, pyridoxal, or pyridoxal phosphate), particularly for pyridoxal.
\end{abstract}

Keywords Green tea · Vitamin B6 · HILIC · Antioxidant activity · DPPH · Isobolographic analysis

\section{Introduction}

Green tea is a worldwide consumed beverage owing to its health-promoting effect caused by the bioactive compounds, such as caffeine, amino acids, and several polyphenols [1,2]. Their popularity recently increased with scientific evidences that demonstrate its beneficial health effects, such as reduced risk of cardiovascular and degenerative diseases, likely due to the antioxidant properties of polyphenols [3, 4]. These compounds exert their antioxidant and antiradical properties by several mechanisms, including direct scavenging of reactive oxygen species, chemical reducing activity, complexation of pro-oxidant metal ions, activation of antioxidant

Electronic supplementary material The online version of this article (https://doi.org/10.1007/s00217-020-03516-w) contains supplementary material, which is available to authorized users.

Aleksandra Sentkowska

sentkowska@slcj.uw.edu.pl

1 Heavy Ion Laboratory, University of Warsaw, Pasteura 5A, 02-093 Warsaw, Poland

2 Department of Chemistry, University of Warsaw, Pasteura 1, 02-093 Warsaw, Poland enzymes, and inhibition of oxidases [5]. It should be mentioned that different kinds of teas have different phenolic profiles, which impact antioxidant capacities, and antibacterial and antiproliferative activities [6]. Recent studies confirmed that green tea catechins inhibit microglial activation which prevents the development of neurological disorders [7]. Catechins have also been shown to play a significant role in cancer prevention and therapy [8]. Recently also epigallocatechin esters are synthesized and studied as potential sources of antioxidants [9]. It needs to be highlighted that green tea as well as green tea catechols cannot replace the standard chemotherapy. Nonetheless, their beneficial effects may support the standard anticancer approach. The growing interest in therapeutic drugs with less or no side effects resulted in discussion about the potential of flavonoids in pain and inflammation [10]. Until now the mechanism of action of flavonoids is not fully understood, but many clinical studies showed their relevance as a potent anti-inflammatory, analgesic and antioxidative group of molecules.

In recent years, dietary supplements and nutraceuticals have received considerable interest due to potential nutritional and therapeutic effects [11]. Nutraceuticals from green tea extracts are sold in different forms, such as pills, capsules, powders, or drinks, and could contain also some 
vitamins or minerals $[12,13]$. They have been used to cure nutritional deficiencies, weight loss, lowering cholesterol levels, and protecting skin from sun damage.

Vitamin B6 is involved in the metabolism of proteins, fats, and carbohydrates as it is an essential cofactor of numerous enzymatic reactions $[14,15]$. This vitamin is also closely linked with the functions of the nervous and immune systems [16]. The recent studies indicate that it can be beneficial as a nutritional supplement as well as a pharmacological agent for disease treatment $[15,17]$. It was demonstrated that pyridoxine, one of the forms of vitamin B6, may scavenge the hydroxyl, peroxyl radicals and thus inhibits lipid oxidation [17].

The aim of this study was the chromatographic separation of different chemical forms of vitamin B6 and catechins in commercially available dietary supplements as well as to evaluate the antioxidant interactions between green tea polyphenols and vitamin B employing scavenging of the 2,2-diphenyl-1-picrylhydrazyl (DPPH) radical. The isobolographic analysis and interaction indexes, both used in pharmacy and phytomedicine to study the interactions between drugs, were used to characterize the potential additive, synergistic, or antagonistic effects [18].

\section{Materials and methods}

\section{Reagents and samples}

The commercial standards of green tea extract (GTE) and vitamins B (pyridoxine, pyridoxal, and pyridoxal phosphate) were purchased from Sigma (Steinheim, Germany). Acetonitrile $(\mathrm{ACN})$, methanol $(\mathrm{MeOH})$, and ethanol $(\mathrm{EtOH})$ were of HPLC grade from Merck (Darmstadt, Germany). Formic acid used for mobile phase preparation was purchased from Merck (Darmstadt, Germany).

Dietary supplements were purchased in a local drugstore in Warsaw. All of them contained vitamin B6 and green tea extracts, other fruit extracts, or bioflavonoids. The supplements used in the study were as follows: Vitalsss Plus Multiwitamina (S1), Vigor UP! (S2), Wita-min plus (S4), which were standard vitamin preparations, Cidrex Plus (S5) supporting weight loss, Climea Forte (S6) and Floradix (S7) dedicated to women during menopause or effort increase, and Skin Beauty (S3) improving skin condition.

\section{Extraction procedure}

Each tablet was milled in a mortar (LLG Labware, Germany) and dissolved in $10 \mathrm{~mL}$ distilled water. The samples were then shaken vigorously for $30 \mathrm{~min}$ and degassed in an ultrasonic bath for $10 \mathrm{~min}$. After filtration through a $0.22 \mu \mathrm{m}$ PTFE filter, they were directly injected into HPLC system.

\section{Antioxidant activity}

The ability to scavenge free radicals by green tea extract was tested using the DPPH radical method previously described by Pyrzyńska and Pękal [19]. The method is based on the reaction between the methanolic solution of DPPH $\left(9 \times 10^{-5} \mathrm{~mol} / \mathrm{L}\right)$ and the sample, mixed in a proper ratio ( $2.4 \mathrm{~mL}$ of radical solution and $0.1 \mathrm{~mL}$ of a sample). In this case, sample means individual components or their mixtures as it is explained in section Isobolographic analysis. The decrease in absorbance was measured $30 \mathrm{~min}$ after mixing at $518 \mathrm{~nm}$ using Lambda 20 Perkin Elmer spectrophotometer.

\section{Instrumentation}

Chromatographic analysis was performed with the Shimadzu LC system consisted of binary pumps LC20-AD, degasser DGU-20A5, column oven CTO-20AC, autosampler SIL20AC, and 8030 triple quadrupole Mass Spectrometer (Shimadzu, Japan) equipped with an ESI source operated in negative or positive ion mode, according to the determined species. The ESI conditions were as follows: the capillary voltage $4.5 \mathrm{kV}$, temperature $400{ }^{\circ} \mathrm{C}$, the source gas flow $3 \mathrm{~L} /$ min, and drying gas flow $10 \mathrm{~L} / \mathrm{min}$. For each compound, the optimum conditions of Multiple Reaction Mode (MRM) were determined (Table 1S.). The quantification of vitamin B6 was done in positive ion mode, while for the catechins the negative ion mode was used. The selection criterion was higher detection sensitivity, which was optimized for each analyte individually.

Compounds were separated on SeQuant ${ }^{\mathrm{TM}}$ ZIC-HILIC column $(100 \times 2.1 \mathrm{~mm}, 3.5 \mu \mathrm{m})$ from Merck (Darmstadt, Germany). For the separation of all studied compounds, mobile phase containing $8 \mathrm{mM}$ formic acid at $\mathrm{pH} 2.8$ (eluent A) and organic solvent ( $\mathrm{ACN}, \mathrm{MeOH}$, or $\mathrm{EtOH}$-eluent B) was used in gradient elution. The gradient profile was as follows: 0-3 $\min 80 \% \mathrm{~B}, 4-7 \mathrm{~min} 50 \% \mathrm{~B}, 8-12 \min 70 \% \mathrm{~B}$, and $13-20 \min 80 \% \mathrm{~B}$. The mobile phase was delivered at $0.2 \mathrm{~mL} / \mathrm{min}$.

\section{Isobolographic analysis}

For characterization of antioxidant effect between GTE and pyridoxine (PE), isobolographic analysis was used. It is based on the construction of line so called isobola. It is a link between the $\mathrm{EC}_{50}$ values obtained for individual studied components. In other words, it is also theoretical additive line. The nature of the interaction between the GTE and PE is determined by the location of the points corresponding to the antioxidant capacity of their mixtures. If the points are located below the isobola curve, the observed effect is 
synergistic, while if they are located above it, the effect is antagonistic. The mixtures of GTE and PE were prepared in concentration ratios as follows: 10:1, 5:1, 2:1, 1:1, 1:2, 1:5, and 1:10. Five different mixtures of GTE and PE in the same concentration ratio was measured. Moreover, each sample was analyzed in triplicate.

\section{Statistical analysis}

The presented results are obtained from at least three independent experiments and presented as average \pm standard deviation. One-way ANOVA and Turkey test were used to determine the difference of means. Significance was defined at $P$ values $<0.05$.

\section{Results and discussion}

For the chromatographic separation of vitamins from B group, usually reversed-phase mode is used. Vitamin B6 was determined in dietary supplements [20,21], vegetables $[22,23]$, or in the urine samples [24] using C18 stationary phase. Pyridoxine was separated from other water-soluble vitamins also using diol column in HILIC mode with 90:10 (w/v) ACN-water containing $5 \mathrm{mM}$ ammonium acetate (pH 5.0) as mobile phase [25]. In this study, HILIC mode with ZIC-HILIC column was applied for the simultaneous determination of different chemical forms of B6 vitamin and catechins. Zwitterionic stationary phase contains two active groups--strongly acidic sulfonic acid group and strongly basic quaternary ammonium group. Both groups are present at 1:1 M ratio. The positive charge is close to the surface, while the negative charge is in the terminal of the bonded ligand and thus is more accessible for the analytes [26]. Water is an essential component of the eluents used in HILIC mode, because it maintains stable layer onto polar stationary phase. Weaker solvent (organic one) is needed to decrease the overall polarity of the whole eluent, and thus, the analytes can be distributed into eluent and water layer. Acetonitrile, methanol, and ethanol were studied as the main components of mobile phases. In general acetonitrile, a polar aprotic solvent is proven to be the best solvent to be used in HILIC mode. Alcohols as methanol, ethanol, or even isopropanol are not widely used; however, some studies showed that they compete for active polar sites on a stationary phase with analytes, whose retention is based on strong hydrogen bonding $[25,27,28]$. Typically the retention time of the analytes increases with the increasing number of carbons in the alcohol molecule [29]. The overall solvent strength of the eluents used in this study is in the order: acetonitrile $<$ ethanol $<$ methanol $<$ water.

The obtained results clearly shows that using alcohol as an organic component of mobile phase is beneficial for the separation, mainly catechin and epicatechin as well as pyridoxine and pyridoxal as shown in Fig. 1. The application of alcohol instead acetonitrile resulted in changing the elution order and increasing the peak intensity. Methanol is known for its strong ability for hydrogen bonding and may disturb the formation of water layer onto polar stationary phase. When it replaces the water molecules in such layer, the analytes with strong hydrogen-bonding potential are less retained [27]. In a comparison to acetonitrile, the use of methanol enhances the signal intensity but did not resolve the problem of coelution. Only the use of ethanol, which lies in the eluotropic series between $\mathrm{ACN}$ and $\mathrm{MeOH}$, allows the analytes to be separated. No significant changes in retention times were observed when methanol was replaced by ethanol. The lack of coelution was the criterion why it was chosen to use an organic component of mobile phase, even if the intensity of the signals were slightly lower than those obtained for methanol.

The optimized composition of the eluent in ZIC-HILIC method was applied for the determination and quantification of catechins and vitamins B6 in commercially available dietary supplements (Table 1). The obtained contents of vitamin B6 are in agreement with the information given by the producer. Catechins were found in all tested supplements; however, only information on their content was placed on the S5 packaging. It should be highlighted that every addition of plant extracts (not only green tea) to the supplement may result in the presence of catechins in it.

The possible interaction between the constituents of dietary supplements or nutraceuticals may enhance or diminish the final antioxidant potential in the mixture [30-33]. The antioxidant interactions (synergic, additive, or antagonistic effects) of green tea extract in combination with vitamin B6

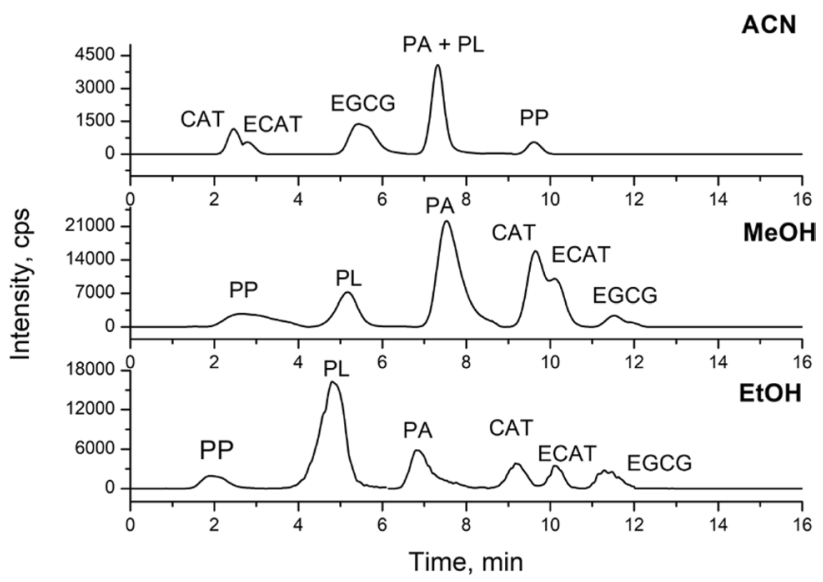

Fig. 1 The impact of organic component of mobile phase on the separation of vitamin B6 and catechins. Gradient elution: $0-3 \min 80 \% \mathrm{~B}$, 4-7 $\min 50 \% \mathrm{~B}, 9-13 \mathrm{~min} 70 \% \mathrm{~B}$, and $13-20 \mathrm{~min} 80 \% \mathrm{~B}$, where $\mathrm{A}$ is $8 \mathrm{mM} \mathrm{HCOOH}(\mathrm{pH} 2.8$ ) and $\mathrm{B}$ is organic component: $\mathrm{ACN}, \mathrm{MeOH}$, or $\mathrm{EtOH}$ 
Table 1 The content of vitamin B6 and catechins in studied dietary supplements

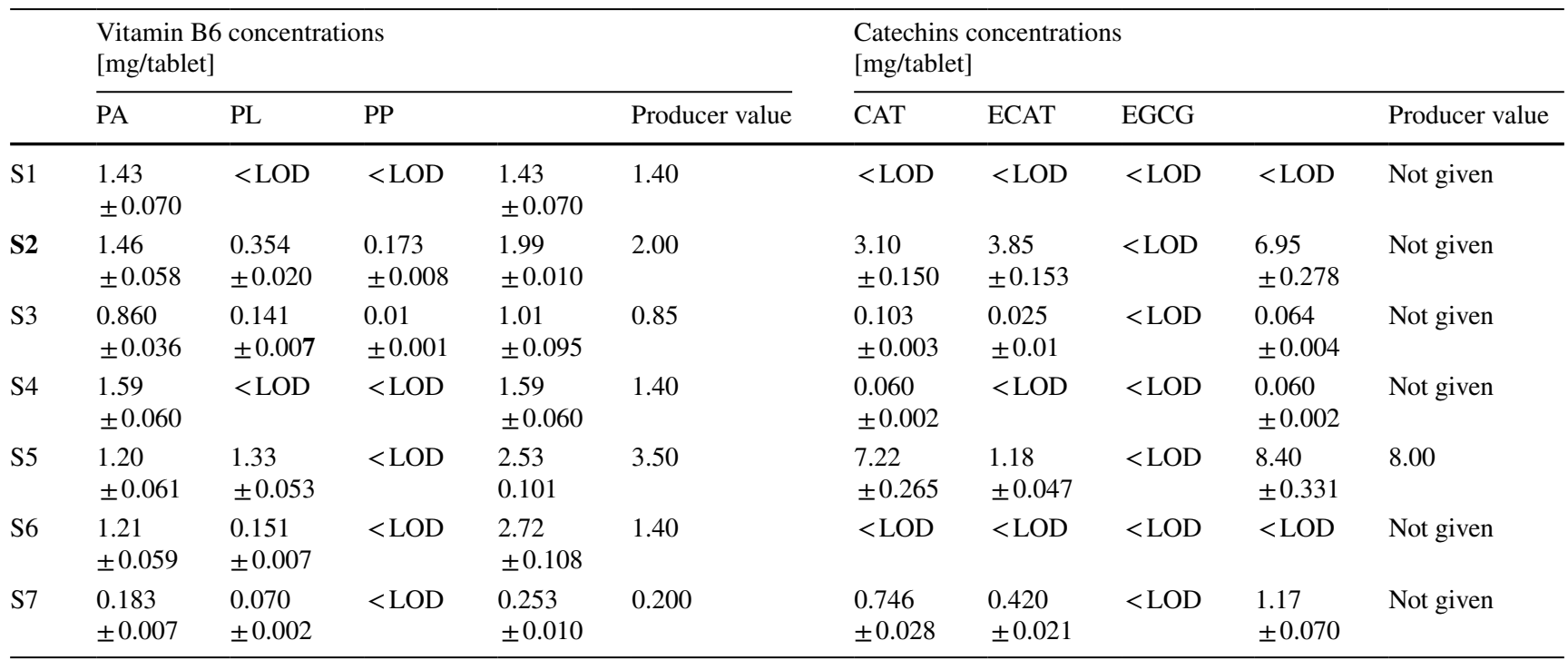

Details regarding samples S1-S7 in experimental section

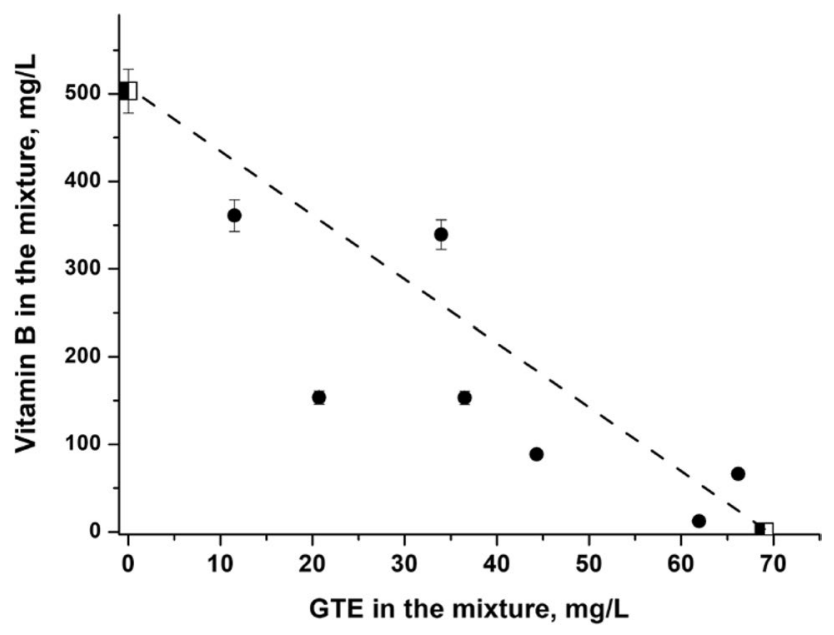

Fig. 2 Isobologram for green tea extract and vitamin B6 in DPPH assay

at different ratios were evaluated using the DPPH method and isobolographic analysis. The isobole method is independent of the mechanism of action and applies under most conditions [17]. The antioxidant activity was expressed in terms of $\mathrm{EC}_{50}$ value, i.e., the concentration (in $\mathrm{mg} / \mathrm{L}$ ) required to scavenge $50 \%$ of DPPH radicals calculated by a linear regression analysis [34]. Seven independent samples (different concentrations of GTE and vitamin B, but the same concentration ratio) were analyzed, and the $\mathrm{EC}_{50}$ values for these mixtures were calculated. The constructed isobola (Fig. 2) shows GTE extracts and B6 vitamin synergistic antioxidant interaction is observed. Exceptions to this trend are points designated for mixtures with a large excess of vitamin or in which GTE and B6 levels in the mixture were equal. These points lie above the additive line. A common theme in the scientific literature is that interactions between antioxidant molecules do occur, but a mechanism that allows a prediction of synergistic and antagonistic interactions is not clear. The kind of interaction depends greatly of the specific antioxidants interacting in the system and the condition behind the evaluation [35-37]. The knowledge about the interaction between polyphenolic compounds and vitamins as well as non-antioxidant compounds is even more poor. Palafox-Carlos reported phenolic acids are capable not only to donate hydrogen atoms to the radical but to donate electrons to regenerate other pro-oxidant phenols [35]. This regeneration mechanism maximizes the AOXC of the system to reduce free radicals. According to Leopoldini et al. [38], phenolic compounds are capable to transfer electrons to other phenolics or antioxidants, promoting their chemical regeneration. It needs to be highlighted that tea is a source not only of catechins but also of other polyphenolics, e.g., polyphenolic acids or flavonoids.

The type of interaction determined on the basis of isobolographic analysis was confirmed by the values of interaction indexes (Table 2). They were calculated according to the equation; $I F=C_{A}+C_{B}$, where $C_{A}$ and $C_{B}$ are the concentrations of the given components in the mixture dividing by its concentration having the same effect as the mixture [30]. The value of interaction index $>1$ indicates synergistic effect; the value $<1$ indicates antagonism, while the value equal to one means additional interactions. Most of the calculated interaction indexes are higher than one. There is no significant differences between the interaction indexes obtained for mixtures, in which GT is in the excess 
Table 2 Interaction indexes for green tea extract and vitamin B6 mixtures

\begin{tabular}{ll}
\hline $\begin{array}{l}\text { Ratio GTE: } \\
\text { vitamin B6 }\end{array}$ & Interaction index \\
\hline $10: 1$ & $0.94 \pm 0.038$ \\
$5: 1$ & $0.92 \pm 0.031$ \\
$2: 1$ & $0.72 \pm 0.025$ \\
$1: 1$ & $1.09 \pm 0.044$ \\
$1: 2$ & $0.82 \pm 0.033$ \\
$1: 5$ & $0.62 \pm 0.023$ \\
$1: 10$ & $1.17 \pm 0.045$ \\
\hline
\end{tabular}

to vitamin B6 (10:1 and 5:1). In such case, antioxidant activity of GT itself is crucial. On the other hand, there is no differences between interaction indexes obtained for the same concentration of both compounds in the mixture (1:1) and the highest concentration of vitamin B6 in comparison to GT (10:1). These two points lie under the isobola (Fig. 1.). Statistically significant effect is observed for the middle concentration range of both studied components. However, based on the values of interaction indexes, all these mixtures showed synergistic effect, which can be used for designing a new dietary supplements. Similar discontinuities, where some points on the isobola departed from the observed trend, were described earlier [31, 32]. Enko and GliszczyńskaŚwigło received three points below the isobola, while the remaining ones indicated an antagonistic effect (position above the isobola) when the interactions between ascorbic acid and green tea were studied by DPPH assay [31]. Such phenomena were not observed when black tea extract was used for the studies. Similar results were obtained when the interactions between green tea and acetaminophen were evaluated [32]. In this case, two points indicated synergistic effect, while other suggested antagonism. Many studies about the antioxidant potential of phenolic compounds in drinks or foods have concluded that it is impossible to predict the antioxidant power of a given product by studying just one type of phenolic compound or other kind of antioxidants contained in the product, such as vitamin $\mathrm{C}$ or E. In some cases, the possible existence of synergistic and antagonistic effects between the various antioxidants present in plant foods and other products have been discussed [39].

Vitamin B6 exists in several forms--pyridoxine, pyridoxal, and pyridoxamine--which differ in a variable group present at one position; pyridoxine carries a hydroxyl group, pyridoxal an aldehyde group, and pyridoxamine has an amino group. All of them are subject to phosphorylation, but pyridoxal phosphate is considered as biologically more active [15]. The effect of the addition of different forms of vitamin B6 to the sample containing $10 \mathrm{mg} / \mathrm{L}$ of green tea extract was evaluated. The kinetic curves of scavenged DPPH radicals by such binary mixtures are presented in Fig. 3. Vitamin B6, regardless of a form, does not react with
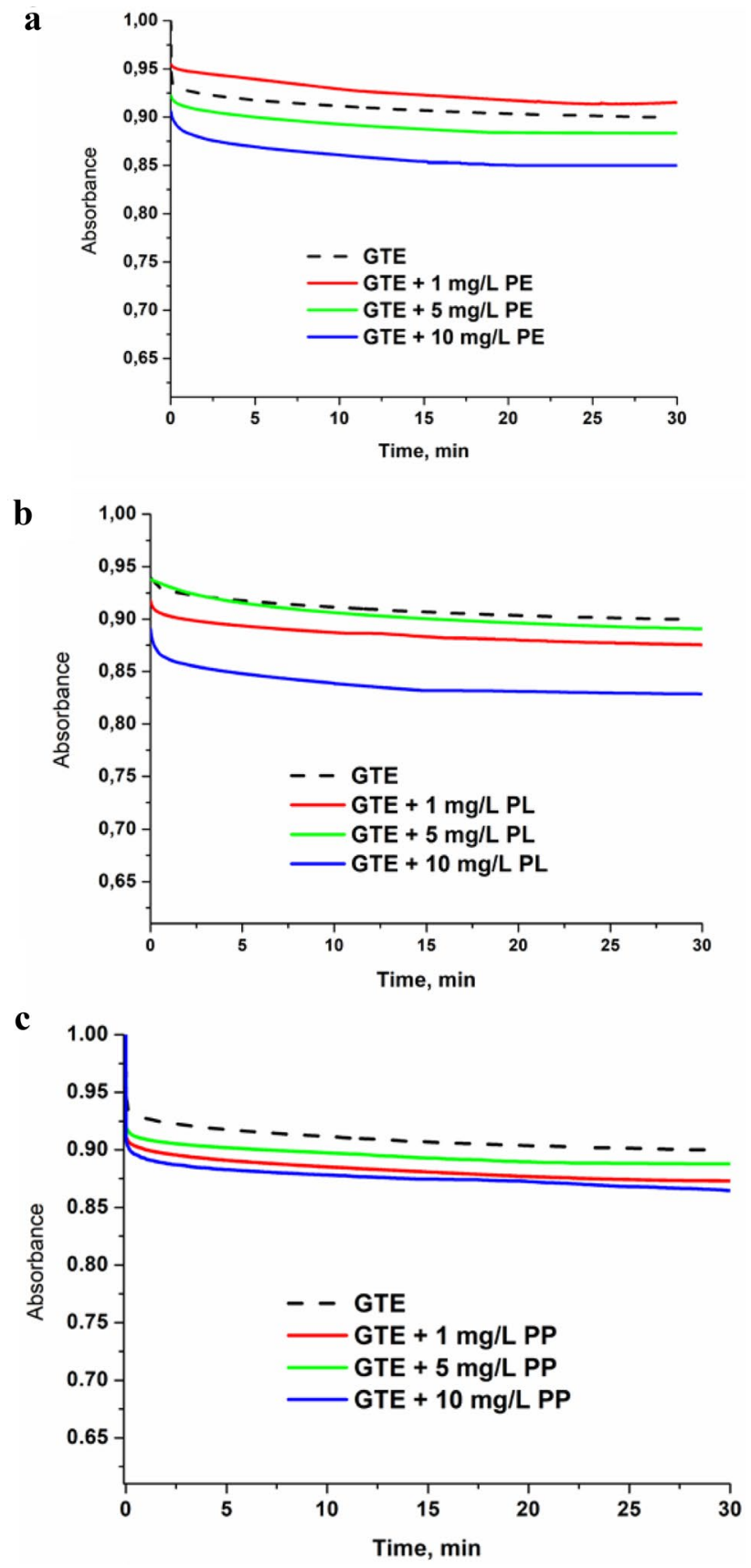

Fig. 3 The kinetic curves of scavenged DPPH radicals by green tea extract (GTE, $10 \mathrm{mg} / \mathrm{L}$ ) and its mixture with different forms of vitamin B6: a pyridoxine (PE); b pyridoxal (PL), and c pyridoxal phosphate (PP)

DPPH radicals, while for GTE, rich in catechins, a relatively fast initial decrease of DPPH absorbance followed by slow subsequent disappearance of this reagent can be observed. Faster DPPH radical bleaching kinetics were observed in the presence of increasing concentration of vitamins B6, particularly for pyridoxal. It suggested that the antioxidant interactions between these two components in mixtures are 
synergistic. For all tested forms of vitamin B6, the highest increase in antioxidant capacity was observed with the highest content of vitamin in the mixture $(10 \mathrm{mg})$. It needs to be highlighted that green tea itself is a mixture of many compounds, so the interactions between the extract and the vitamin are complex. Many well-documented clinical reports of herb-drug interaction are described in the literature [40-44]. Such type of interaction may be more common than simple drug-drug interaction and more complex because herbal infusions contain multiple active ingredients. According to Mouly [40], the ingredients of herbs can alter drug absorption, distribution, and metabolism. On the other hand, this effect has an impact on the antioxidant capacity of the herbal infusion. The described interactions can be referred to the interactions of ingredients present in green tea with vitamin B6. The observed differences in the interaction of tea with different forms of vitamin may be the result of differences in the structure of the molecule of individual forms of B6 vitamin. Several hypotheses have been postulated to explain synergy and antagonism in the antioxidant response $[41,42]$. These effects generally depend on effective concentrations of the compounds, their polarity, as well as the reaction rate. More knowledge on these interactions and a deep understanding of their driving forces should lead to a better understanding of formulation strategies for improved delivery of bioactive compounds.

Green tea extracts containing a great source of antioxidants are very popular as food supplements. One of them, S5 (Cidrex Plus), according to its label contains $20 \mathrm{mg}$ of GTE and $3.5 \mathrm{mg}$ vitamin B6 (as pyridoxine). Figure 1S shows the kinetic curves of scavenged DPPH radicals by the content of S5 (Cidrex) capsule with pyridoxine additives. As can be seen, the mixtures with increasing amounts of vitamin B exhibited higher antioxidant activity in DPPH assay than this capsule. However, this increase is dose dependent. Additionally, the presence of other components in that food supplement may have some influence of measured value of antioxidant activity.

\section{Conclusions}

These studies demonstrate the potential of application of ethanol as an organic component of mobile phase in HILIC mode in the separation of different chemical forms of vitamin B6 and catechins. The influence of vitamin B on the antioxidant activity of green tea extract using DPPH assay was also shown. These results may be important from the point of view of supplementation. However, more studies are needed for better describing the catechins-vitamin B antioxidant interactions.

\section{Compliance with ethical standards}

Conflict of interest The authors have no conflict of interest to disclose.

Compliance with ethics requirements This study does not involve any human or animal testing.

Open Access This article is licensed under a Creative Commons Attribution 4.0 International License, which permits use, sharing, adaptation, distribution and reproduction in any medium or format, as long as you give appropriate credit to the original author(s) and the source, provide a link to the Creative Commons licence, and indicate if changes were made. The images or other third party material in this article are included in the article's Creative Commons licence, unless indicated otherwise in a credit line to the material. If material is not included in the article's Creative Commons licence and your intended use is not permitted by statutory regulation or exceeds the permitted use, you will need to obtain permission directly from the copyright holder. To view a copy of this licence, visit http://creativecommons.org/licenses/by/4.0/.

\section{References}

1. Namal Senanayake SPJ (2013) Green tea extract: chemistry, antioxidant properties and food applications-A review. J Funct Foods 5:1529-1541. https://doi.org/10.1016/j.jff.2013.08.011

2. Maiti S, Nazmeen A, Medda N, Paqtra R, Ghosh TK (2019) Flavonoids green tea against oxidant stress and inflammation with related human diseases. Clin Nutr Exp 24:1-14. https://doi. org/10.1016/j.yclnex.2018.12.004

3. Costa C, Tsatsakis A, Manoulakis C, Teodoro M, Brigugilio G, Caruso E, Tsoukalas D, Margina D, Dardiotis E, Kouretas D, Fenga C (2017) Current evidence on the effect of dietary polyphenols intake on chronic diseases. Food Chem Toxic 110:286-299. https://doi.org/10.1016/j.fct.2017.10.023

4. Mancini E, Beglinger C, Dreweb J, Zanchia D, Undine E, Langa UE (2017) Green tea effects on cognition, mood and human brain function: a systematic review. Phytomed 34:26-37. https://doi. org/10.1016/j.phymed.2017.07.008

5. Grzesik M, Naparło K, Bartosz G, Sadowska-Bartosz I (2014) Antioxidant properties of catechins: comparison with other antioxidants. Food Chem 241:480-492. https://doi.org/10.1016/j. foodchem.2017.08.117

6. Cardoso RR, Neto RO, D'Almeida CTS, Nascimento TP, Pressete CG, Azevedo L, Martino HSD, Cameron LC, Ferreira MSL, deBarros FAR (2020) Kombuchas from green and black teas have different phenolic profile, which impacts their antioxidant capacities, antibacterial and antiproliferative activities. Food Res Int 128:108782

7. Farkhondeh T, Pourbagher-Shahri AM, Ashrafizadeh M, Folgado SL, Rajabpour-Sanati A, Khazdair MR, Samarghandian S (2020) Green tea catechins inhibit microglial activation which prevents the development of neurological disorders. Neural Reg Res 15:1792-1798

8. Musial C, Kuban-Jankowska A, Górska- Ponikowska M (2020) Beneficial properties of green tea catechins. Int J Mol Sci 21:1744-1755

9. Ambigaipalan P, Oh WY, Shahidi F (2020) Epigallocatechin (EGC) esters as potential sources of antioxidants. Food Chem 309:125609

10. Ferraz CR, Carvalho TT, Manchope MF, Artero NA, RasquelOliviera FS, Fattori V, Casagrande R, Verri WA Jr (2020) Therapeutic potential of flavonoids in pain and inflammation: mechanism of action, pre-clinical and clinical data and pharmaceutical development. Molecules. https://doi.org/10.3390/molecules25030762 
11. Nasri H, Baradaran B, Shirzad H, Rafieian-Kopaei M (2014) New concepts in nutraceuticals as alternative for pharmaceutical. Inter J Prev Med 5:1487-1499 (PMCID: PMC4336979)

12. Patel S (2013) Green tea as a nutraceutical: the latest development. Food Sci Techn Res 19:923-932. https://doi.org/10.3136/ fstr. 19.923

13. Jia W, Chu X, Chang J, Zhang F (2015) High-throughput screening of vitamins and natural antioxidants in nutraceuticals from green tea extracts by liquid chromatography coupled to quadrupole orbitrap mass spectrometry. J Chromatogr A 1406:337-341. https://doi.org/10.1016/j.chroma.2015.06.050

14. Hellman H, Mooney S (2010) Vitamin B6: a molecule for human health ? Molecules 15:442-459. https://doi.org/10.3390/molec ules 15010442

15. Mooney S, Hellman H (2010) Vitamin B6: killing two birds with one stone? Phytochem 71:495-501. https://doi.org/10.1016/j. phytochem.2009.12.015

16. Taş S, Sarandöl E, Drican M (2014) Vitamin B6 supplementation improves oxidative stress and enhances serum paraoxonase/ arylesterase activities in streptozoticin-induced diabetic rats. Sci World J. https://doi.org/10.1155/2014/351598

17. Danielyan KE, Simonyan AA (2017) Protective abilities of pyridoxine in experimental oxidative stress settings in vivo and in vitro. Biomed Pharmacotherap 86:537-540. https://doi. org/10.1016/j.biopha.2016.12.053

18. Williamson EM (2001) Synergy and other interactions in phytomedicines. Phytomed 8:401-409. https://doi. org/10.1078/0944-7113-00060

19. Pyrzynska K, Pękal A (2013) Application of free radical diphenylpicrylhydrazyl (DPPH) to estimate antioxidant capacity of food samples. Anal Meth 55:4288-4295. https://doi.org/10.1039/ C3AY40367J

20. Heudi O, Kilinc T, Fontannaz P (2005) Separation of watersoluble vitamins by reversed-phase high performance liquid chromatography with ultra-violet detection: application to polyvitaminated premixes. J Chromatogr A 1070:49-56. https://doi. org/10.1016/j.chroma.2005.02.033

21. Chen Z, Chen B, Yao S (2006) High-performance liquid chromatography/electrospray ionization-mass spectrometry for simultaneous determination of taurine and 10 water-soluble vitamins in multivitamin tablets. Anal Chim Acta 569:169-175. https://doi. org/10.1016/j.aca.2006.03.099

22. Kall MA (2003) Determination of total vitamin B6 in foods by isocratic HPLC a comparison with microbiological analysis. Food Chem 82:315-327. https://doi.org/10.1016/S0308-8146(02)00568 $-\mathrm{X}$

23. Santos J, Mendiola JA, Oliviera MB, Ibanez E, Herrero M (2012) Sequential determination of fat- and water-soluble vitamins in green leafy vegetables during storage. J Chromatogr A 1261:179188. https://doi.org/10.1016/j.chroma.2012.04.067

24. Cho CM, Ko JH, Cheong WJ (2000) Simultaneous determination of water-soluble vitamins excreted in human urine after eating an overdose of vitamin pills by HPLC method coupled with a solid phase extraction. Talanta 51:799-806. https://doi.org/10.1016/ s0039-9140(00)00283-6

25. Karatapanis AE, Fiamegos YC, Stalikas CD (2010) Study of behaviour of water-soluble vitamins in HILIC on a diol column. Chromatogr 71:751-759. https://doi.org/10.1365/s1033 7-010-1564-3

26. Sentkowska A, Pyrzynska K (2018) Zwitterionic hydrophilic interaction liquid chromatography coupled to mass spectrometry for analysis of beetroot juice and antioxidant interactions between its bioactive compounds. LWT_Food Sci Tech 93:641-648. https ://doi.org/10.1016/j.lwt.2018.04.023

27. Hao Z, Xiao B, Wang N (2008) Impact of column temperature and mobile phase components on selectivity of hydrophilic interaction chromatography (HILIC). J Sep Sci 31:1449-1464. https://doi. org/10.1002/jssc. 200700624

28. Li R, Hunag J (2004) Chromatographic behaviour of epirubicin and its analogues on high-purity silica in hydrophilic interaction chromatography. J Chromatogr A 1041:163-169. https://doi. org/10.1016/j.chroma.2004.04.033

29. Hemstrom P, Irgum $K$ (2006) Hydrophilic interaction chromatography. J Sep Sci 29:1784-1821. https://doi.org/10.1002/jssc.200600199

30. Wang S, Meckling KA, Marcone MF, Kakuda Y, Tsao R (2011) Synergistic, additive and antagonistic effects of food mixtures on total antioxidant capacities. J Agric Food Chem 59:960-968. https ://doi.org/10.1021/jf1040977

31. Enko J, Gliszczyńska-Świgło A (2015) Influence of the interactions between tea (Camelia sinensis) extracts and ascorbic acid on their antioxidant activity: analysis with interaction indexes and isobolograms. Food Add Contam Part A 32:1234-1242. https:// doi.org/10.1080/19440049.2015.1049218

32. Sentkowska A, Pyrzyńska K (2018) Investigation of antioxidant interaction between green tea polyphenols and acetaminophen using isobolographic analysis. J Pharm Biomed Anal 159:393-397

33. Durak A, Gawlik-Dzik U, Kowalska J (2015) Coffee with gingerInteractions of biologically active phytochemicals in the model system. Food Chem 166:261-269. https://doi.org/10.1016/j.foodc hem.2014.06.075

34. Sentkowska A, Pyrzynska K (2018) Investigation of antioxidant interaction between green tea polyphenols and acetaminophen using isobolographic analysis. J Pharm Biom Anal 159:393-397. https://doi.org/10.1016/j.jpba.2018.07.029

35. Sebaugh JL (2011) Guidelines for accurate EC50/IC50 estimation. Pharm Stat 10:128-134. https://doi.org/10.1002/pst.426

36. Palafox-Carlos H, Gil-Chaves J, Sotelo-Mundo SS, Namieśnik J, Gorinstein S, Gonzales-Aguilar GA (2012) Antioxidant interaction between major polyphenolic compounds found in "Atafulo' mango pulp: chlorogenic, gallic, protocatechuic and vanillic acids. Molecules 17:12657-12664

37. Rebber JD, Egget DL, Parker TL (2011) Antioxidant capacity interactions and and a chemical/structural model of phenolic compounds found in strawberries. Int J Food Sci Nutr 62:445-452

38. Leopoldini M, Marino T, Russo N, Toscano M (2004) Antioxidant properties of phenolic compounds: $\mathrm{H}$-atom versus electron transfer mechanism. J Phys Chem A 108:4916-4922

39. Garcia-Alonso M, Rimbach G, Sasai M, Nakahara M, Matsugo S, Uchida Y, Rivas- Gonzalo JC, De Pascual-Teresa S (2005) Electron spun resonance spectroscopy studies on the free radical scavenging activity of wine anthocyanins and pyranoanthocyanins. Mol Nutr Food Res 49:1112-1119

40. Posadzki P, Watson L, Ernst E (2012) Herb-drug interactions: an overview of systematic reviews. Br J Clin Pharmacol 75:603-618

41. Mouly S, Lioret-Linares C, Sellier PO, Sene D, Bergmann JF (2017) Is the clinical relevance of drug-food and drug-herb interactions limited to grapefruit juice and Saint-John's Worth? Pharmacol Res 118:82-92

42. Izzo AA, Hoon-Kim S, Radhakrishnan R, Williamson EM (2016) A critical approach to evaluating clinical efficacy, adverse events and drug interactions of herbal remedies. Phytoher Res 30:691-700

43. Prieto MA, Murado MA, Vázquez JA (2014) Quantification, characterization and description of synergy and antagonism in the antioxidant response. Food Res Intern 60:218-229. https://doi. org/10.1016/j.foodres.2013.09.033

44. Albassam AA, Markowitz JS (2017) An appraisal of drug-drug interactions with green tea (Camellia sinensis). Planta Med 83:496-508. https://doi.org/10.1055/s-0043-100934

Publisher's Note Springer Nature remains neutral with regard to jurisdictional claims in published maps and institutional affiliations. 